\title{
The evolution of monogamy in humans
}

\section{Personal view}

Sexual behaviours are likely to have evolved under strong selective pressures since they are directly related to the breeding success of individuals.

It is striking that the sexual behaviour of humans differs so markedly from that of the two chimpanzee species with which we share more than $99 \%$ of our genome. The behaviour of the less closely related gorilla is more akin to that of many mammals-for example, ungulates/sea elephants, in which sexually dimorphic dominant males herd smaller females into groups over which they maintain copulatory monopoly. Still more distantly related apes-for example, gibbons, are not sexually dimorphic and are monogamous.

Both humans and chimpanzees display sexual dimorphism which inclines sociobiologists to the uncomfortable view that the human's "natural" sexual behaviour may be not dissimilar from that of the chimpanzee. However, there are major differences in chimp and in human sexuality. The relative size of the penis and the relative time spent during copulation are described by Diamond ${ }^{1}$ but these may have evolved through other mechanisms. The newborn human infant, for example, is relatively much larger than that of the chimp.
Another difference is that human copulation is almost universally conducted in private, in contrast with that of the chimpanzee.

Jane Goodall, during her prolonged study of Pan troglodytes in the wild, ${ }^{2}$ observed several episodes of copulation which did occur in privacy. Subordinate males (who would not have mating rights with females in oestrus in the open social group) would coax a partner (preferably one who was just about to go into oestrus) away from the group so as to be able to monopolise her. This "sequestering" of female by male chimpanzees has been observed by others. ${ }^{3}$ Other aspects of human as opposed to simian sexual behaviour may have coevolved from this strategy.

HUMPHREY BIRLEY

Department of Medical Microbiology and Genito-urinary Medicine, Royal Liverpool University Hospital, Prescot Street, Liverpool L7 8XP

ADRIAN RENTON

Department of Social Science and Medicine,

Imperial College School of Medicine, London SW6 $1 R Q$

Diamond J. The rise and fall of the third chimpanzee. London: Hutchinson Radius, 1993.

2 Goodall J. Through a window. London: Weidenfield and Nicholson, 1990.

3 Symons D. The evolution of human sexuality. Oxford: Oxford University Press, 1979. 